\title{
COMET HAZARD TO THE EARTH
}

\author{
S. I. Ipatov \\ Institute of Applied Mathematics, Miusskaya sq.4, Moscow 125047, Russia; ipatov@keldysh.ru
}

\begin{abstract}
'Migration of trans-Neptunian objects to the Earth is considered. Due to the gravitational influence of large trans-Neptunian objects and mutual collisions, some objects can get such orbits, from which they can be moved inside the Solar System under the gravitational influence of planets. About 10-20\% or even more '1-km Earth-crossers could have come from the Edgeworth-Kuiper belt and can move in Jupiter-crossing 'orbits. The number of former trans-Neptunian objects that cross the Earth's orbit moving in orbits with aphelia inside Jupiter's orbit can be of the same order of magnitude. A total mass of icy planetesimals 'delivered to the Earth during formation of the giant planets can be about the mass of the Earth's oceans.
\end{abstract}

Keywords: trans-Neptunian objects, near-Earth objects, migration

Paper submitted to "Advances in Space Research" (in press). 


\section{INTRODUCTION}

Near-Earth objects (NEOs), i.e., objects with perihelion distance $q \leq 1.3 \mathrm{AU}$ and aphelion distance $Q \geq 0.983 \mathrm{AU}$, are considered to have a chance to collide the Earth in some future. There are more than 30 NEOs larger than $5 \mathrm{~km}$ in diameter, about 1500 NEOs larger than $1 \mathrm{~km}$, and 135,000 larger than 100 $\mathrm{m}$ (Rabinowitz et al., 1994). About half of NEOs are Earth-crossers. It is considered that diameter of the object that caused the Tunguska event in 1908 was about 60-70 m. The main sources of NEOs are considered to be the main asteroid belt and the Edgeworth-Kuiper belt (EKB). Some Earth-crossers are long-period comets, which came from the Oort cloud located at a distance $(2-3) \cdot 10^{4}-10^{5}$ AU from the Sun. It is not yet clear, how many NEOs came from the EKB. Some scientists (Farinella et al., 1993) considered that most of meteorites and NEOs are asteroidal fragments, other (Wetherill, 1988) supposed that half of NEOs are former short-period comets, and Eneev (1980) considered that most NEOs came from the EKB. A cometary origin of NEOs, as well as meteorites, was suggested by Öpik (1963). Fernandez (1980) first supposed that short-period comets came from the trans-Neptunian belt. Icy bodies can also migrate inside the Solar System from the regions located between the EKB and the Oort cloud. According to Weissman (1995), the dynamically inactive region beyond 45 AU may extend out to $1000 \mathrm{AU}$ or even more and contain up to several times $10^{13}$ objects with a total mass of several hundred Earth masses.

Some bodies leave the main asteroid belt via different mean motion and secular resonances (Gladman et al., 1997). Objects that came from the main asteroid belt got in the resonant regions, from which they were delivered to a near-Earth space, mainly via collisions with other asteroids (for small asteroids also due to the Yarkovsky effect). Ipatov (1995b) obtained that only a few percents of asteroids could get into the main resonances with Jupiter $(3 / 1,5 / 2,7 / 3$, and 2/1) during the last 4 Gyr due to the mutual gravitational influence of asteroids. Migliorini et al. (1998), Morbidelli and Nesvorny (1999) showed that mean motion resonances with Mars $(3 / 5,7 / 12,4 / 7,5 / 9,7 / 13$, and 1/2), mean motion resonances with Jupiter $(7 / 2$ and 10/3), and three-body mean motion resonances between Jupiter, Saturn, and the asteroid, or Mars, Jupiter, and the asteroid could deliver material to Mars and Earth. As the number of resonances delivering bodies to the Earth is not small, even small variations in semimajor axes can put some asteroids in the resonances. So the role of mutual gravitational influence in transportation of asteroids to the Earth may not be very small. According to Wetherill (1988) and Weissman et al. (1989), it is difficult to explain the number of NEOs and features of their orbits (for example, their mean inclinations, which are larger than those in the main asteroid belt), if one considers only asteroidal sources. Wetherill (1991) considered that NEOs should come from the trans-Neptunian belt, but not from the Oort cloud in order to supply present inclinations of orbits of NEOs. To our opinion, the mean inclination of those NEOs that came from the main asteroid belt can be the same as that of all NEOs $\left(15^{\circ}\right)$ and can be larger than the mean inclination of main-belt asteroids $\left(10^{\circ}\right)$, because these objects could increase their inclinations when they were in resonances.

In April 2001, 369 typical trans-Neptunian objects (TNOs) and 67 Centaurs/SDOs were discovered. Typical TNOs are often called EKB objects (EKBOs). EKBOs have semimajor axes between 35 and 50 $\mathrm{AU}$, and their eccentricities are less than 0.4. As there is no exact boundary between Centaurs and SDOs (scattered disk objects), these objects are listed in one table. Most of SDOs have $a>50$ AU. The largest TNO (2000 WR106, i.e., Varuna (20000), $a=43.3 \mathrm{AU}, e=0.056, i=17^{\circ}$ ) has diameter about $1000 \mathrm{~km}$. Most of other TNOs has diameters $d \sim 100-400 \mathrm{~km}$. About 70,000 of such sized bodies were inferred (Jewitt et al. 1996, Levison and Duncan 1997). The total mass of EKBOs $M_{E K B}$ is estimated to be about $0.06 m_{\oplus}$ to $0.25 m_{\oplus}$, where $m_{\oplus}$ is the mass of the Earth (Jewitt et al., 1996). The total number of bodies having diameter $d \geq 1 \mathrm{~km}$ within the $30 \leq a \leq 50 \mathrm{AU}$ is estimated to be about $10^{10}$ (Jewitt et al., 1996), $10^{11}$ (Jewitt, 1999), or $5 \cdot 10^{9}$ (Jewitt and Fernandez, 2001). The mean values of eccentricity and inclination for first 181 TNOs (with $a \leq 50 \mathrm{AU}$ ) are equal to 0.09 and 8.1 ${ }^{\circ}$, respectively. For 34 SDOs with $a>50 \mathrm{AU}$ and $q \geq 30 \mathrm{AU}$, these values were 0.5 and $15^{\circ}$. A total mass of SDOs that move in eccentric orbits between 40 and $200 \mathrm{AU}$ is estimated to be equal to $0.5 M_{\oplus}$ (Luu et al., 1997) or to 0.05 $M_{\oplus}$ (Trujillo et al., 2000). In the latter paper the number of "scattered" objects with $d>100 \mathrm{~km}$ is considered to be about $3 \cdot 10^{4}$. 
Long-period and sungrazing comets also deliver material to the terrestrial planets. About $80 \%$ of all known comets are long-period comets, more than 80 members of the family being sungrazing comets. More than half of the close (up to $0.102 \mathrm{AU}$ ) encounters of comets with the Earth belong to long-period comets (http://cfa-www.harvard.edu/iau/lists/ClosestComets.htm). Thus, the number of collisions of active longperiod comets with the terrestrial planets may be of the same order of magnitude as that for active shortperiod comets, but short-period comets supply a larger number of extinct comets. Some long-period comets are as large as the Hale-Bopp comet, which had diameter of about $30 \mathrm{~km}$, perihelion distance $q=0.914$ AU and moved almost perpendicular to the plane of the Earth's orbit. The mean impact probability of a long-period comet with the Earth per revolution is estimated as $(2-3) \cdot 10^{-9}$ (Marsden and Steel, 1994).

Active and extinct periodic comets may account altogether for about $20 \%$ of the production of terrestrial impact craters larger than $20 \mathrm{~km}$ in diameter (Shoemaker et al., 1994). About 40 active and 800 extinct Earth-crossing Jupiter-family comets with period $P<20 \mathrm{yr}$ and nuclei $\geq 1 \mathrm{~km}$, and about 140-270 active Earth-crossing Halley-family comets $(20<P<200$ yr) were estimated by Shoemaker et al., some of the latter still assumed to come from the Oort cloud. The study of dynamical evolution of Halley-type comet orbits for about \pm 1 Myr showed that some of them got into Earth-crossing orbits for $1 / 3$ to $2 / 3$ of the considered time interval (Bailey and Emel'yanenko, 1996). The probabilities of collisions of short-period comets with Earth and Venus were estimated to be quite similar, whereas that with Mars is an order of magnitude smaller (Nakamura and Kurahashi, 1998). About 1\% of observed Apollos cross Jupiter's orbit (and additional 1\% of Apollos have aphelia between 4.7-4.8 AU), but these Jupiter-crossers move far from the Earth during most of time, so their actual portion among ECOs is greater than that for observed objects. The portion of Earth-crossers among observed Jupiter-family comets is about $10 \%$.

Beside comets and asteroids, numerous meteoroid-like bodies impact the terrestrial planets. About 98-99\% of such bodies with masses less than $100 \mathrm{~g}$ in the vicinity of the Earth are assumed to be of cometary origin, and, in particular, the orbit of the Geminid meteor stream nearly coincides with the orbit of asteroid 3200 Phaethon (Whipple, 1983). Obviously, spectral variations in the collection of meteorites are considerably wider than those of asteroids (Britt et al., 1992). Note that water was found in the Monahans (1998) H5 chondrite (Zolensky et al., 1999) and it could exist in some other meteorites (Vilas and Zolensky, 1999). Morbidelli et al. (2000) argues that during the formation of the main asteroid belt the bodies that migrated from this belt could deliver to the Earth even more water than comets.

From the investigation of lunar craters, Hartmann (1995) considers for a relatively uniform size distribution of interplanetary impactors of mixed origins back to 4 Gyr ago. The frequency of Earth impact by NEOs is estimated to be larger by a factor 2, 14, 24 and 30 as compared to Venus, Mars, Moon and Mercury, respectively (Bottke et al., 1994). Shoemaker et al. (1990) showed that asteroidal impacts probably dominated the production of craters on the Earth with diameter $D_{c r}<30 \mathrm{~km}$, whereas cometary impacts were responsible for the craters with $D_{c r}>50 \mathrm{~km}$. This feature can be explained by the idea that TNOs can leave their residence EKB region essentially without collisions, in contrast to main belt asteroid bodies that experienced multiple collisions (Ipatov, 1998, 1999).

In this paper we consider the migration of TNOs and icy planetesimals to the Earth.

\section{EARLY MIGRATION OF ICY BODIES}

\section{Formation of the Giant Planets and Trans-Neptunian Objects}

Estimates of the number of comets migrated to the Earth during the formation of the giant planets depend on the model of their formation. Such bombardment culminated from 4.5 to 4 Gyr ago. It is generally agreed that the total mass of planetesimals in the feeding zone of the giant planets exceeded by a factor of several the total mass of solids that entered into the giant planets (Safronov, 1972). The total mass $M_{U N}$ of planetesimals in the feeding zone of Uranus and Neptune could exceed $100 m_{\oplus}$ (Ipatov, 1987, 1993). Most of these planetesimals could still move in this zone when Jupiter and Saturn had accreted the bulk of their masses. Zharkov and Kozenko (1990) suggested that Uranus and Neptune accreted a considerable 
portion of their masses in the feeding zone of Saturn, where they aquired also hydrogen envelopes. In a series of numerical experiments, Ipatov $(1991,1993)$ showed that the embryos of Uranus and Neptune could increase their semimajor axes from $\leq 10 \mathrm{AU}$ to their present values, moving permanently in orbits with small eccentricities, due to the gravitational interactions with the planetesimals that migrated from beyond $10 \mathrm{AU}$ to Jupiter. Later on, the idea of a narrow zone, where all giant planets have been formed, was supported and further developed by Thommes et al. (1999). In my opinion, several giant planets moving in close orbits could be formed around another star, but some of these planets, due to their mutual gravitational interactions, could be ejected into hyperbolic orbits and the remaining extrasolar planets (planet) could get large eccentricities. Ipatov $(1987,1993)$ showed that during the accumulation of the giant planets the planetesimals with the total mass of several tens $m_{\oplus}$ entered the trans-Neptunian region. These planetesimals could increase the eccentricities of 'local' TNOs and swept most of these TNOs, which total initial mass could exceed $10 m_{\oplus}$. A small part of such planetesimals could left beyond Neptune's orbit in highly eccentric orbits such as those of SDOs.

Stern (1995, 1996a,b), Stern and Colwell (1997), Davis and Farinella (1997), Kenyon and Luu (1998, 1999) investigated the formation and collisional evolution of the EKB. In their models, the process of accumulation of TNOs took place at small (usually about 0.001) eccentricities and a massive belt. To our opinion, due to the gravitational influence of forming giant planets (our runs showed that maximal eccentricities of TNOs always exceeded 0.05 during $20 \mathrm{Myr}$ under the gravitational influence of the giant planets) and the mutual gravitational influence of planetesimals, such eccentricities may not exist during all the time needed for the accumulation of TNOs, though gas drag could decrease eccentricities and the gravitational influence of forming planets could be less. Eneev (1980) supposed that large TNOs were formed from rarefied dust-gas condensations. We consider that TNOs with $d \geq 100 \mathrm{~km}$ moving now in not very eccentric orbits could be formed directly by the compression of large local rarefied condensations, but not by the accretion of smaller solid planetesimals. Probably, some largest asteroids and planetesimals with $d \geq 100 \mathrm{~km}$ in the zone of the giant planets could be formed in the same way. Some smaller objects can be debris of larger objects and other small objects could be formed directly by compression of condensations. Even if at some instant of time at approximately the same distance from the Sun, the dimensions of initial condensations, which had been formed from the dust layer due to gravitational instability, had been almost identical, there was a distribution in masses of the final condensations, which compressed into the planetesimals. As in the case of accumulation of planetesimals, there could be a "run-away" accretion of condensations.

\section{Early Impactor Delivery}

The total mass of the planetesimals from the feeding zone of Uranus and Neptune that collided with Earth from orbits crossing both the orbits of Jupiter and Earth can be estimated by the following formula: $m_{E c}=M_{U N} p_{J} p_{J E} \Delta t_{E} / T_{E}$, where $p_{J}$ is the fraction of objects from the feeding zone of Uranus and Neptune that reached Jupiter's orbit; $p_{J E}$ is the fraction of Jupiter-crossing objects that reached the orbit of Earth during their lifetimes; $\Delta t_{E}$ is the mean time during which a Jupiter-crossing object crosses the orbit of Earth; and $T_{E}$ is the characteristic time for such an object to impact the Earth. Let us assess quantitatively the factors of the above formula. An estimate of Jupiter-crossing objects can be made on the basis of the orbital evolution of comet $\mathrm{P} / 1996 \mathrm{R} 2$ (Lagerkvist) having the parameters $\left(a \approx 3.79 \mathrm{AU}, e \approx 0.31, i \approx 2.6^{\circ}\right)$. This results in $p_{J E} \approx 0.2$ and $\Delta t_{E} \approx 5 \cdot 10^{3} \mathrm{yr}$ (Ipatov and Hahn, 1999). Note that the mean inclination of Earth-crossing objects is about $15^{\circ}$ and that the larger $a$, the larger $T_{E}$. At $e=0.7, a=3.06 \mathrm{AU}$, and $i$ varing from 0 to $30^{\circ}$, we have $T_{E}=400 \mathrm{Myr}$. Now, taking $M_{U N}=100 m_{\oplus}, p_{J E}=0.2, p_{J}=0.5$, $\Delta t_{E}=5 \cdot 10^{3} \mathrm{yr}$, and $T_{E}=400 \mathrm{Myr}$, we obtain $m_{E c}=1.2 \cdot 10^{-4} m_{\oplus}$. For $\Delta t_{E}=10^{4} \mathrm{yr}$, the value of $m_{E c}$ is greater by a factor 2, both figures being comparable with the mass of water in the Earth's oceans (about $2 \cdot 10^{-4} m_{\oplus}$ ). The above quite rough estimates were obtained for the planetesimals which came from the feeding zone of Uranus and Neptune and then cross both the orbits of Jupiter and Earth. The same order of $m_{E c}$ can be obtained for the planetesimals migrated from the feeding zones of Jupiter and Saturn. 
Probably, even more former planetesimals from the feeding zone of the giant planets could impact the Earth from Encke-type orbits with aphelia $Q \leq 4.5$ AU. Some objects initially formed beyond 30 AU could also migrate to the Earth during planet formation.

\section{PRESENT MIGRATION OF COMETS FROM THE EDGEWORTH-KUIPER BELT TO THE EARTH \\ Objects Leaving the Edgeworth-Kuiper Belt}

Under the gravitational influence of the giant planets, some TNOs can increase their eccentricities, begin to cross Neptune's orbit, and leave the EKB (Torbett and Smoluchowski, 1990; Gladman and Duncan, 1990; Holman and Wisdom, 1993; Levison and Duncan, 1993; Duncan et al., 1995; Duncan and Levison, 1997; Levison and Duncan, 1997). As for the main asteroid belt (MAB), some TNOs can get orbits, which become planet-crossing after some time, due to collisions. Let us compare the rate of collisions in the MAB and EKB. There are about $10^{6}$ main-belt asteroids with diameter $d \geq 1 \mathrm{~km}$; the number of asteroids with $d \geq d_{*} \geq 1 \mathrm{~km}$ is proportional to $d_{*}^{-\alpha}$, with $\alpha$ between 2 and 2.5 (Binzel et al., 1991; Hughes and Harris, 1994). The mass ratio of an asteroid and an impactor for which catastrophic destruction occurs is about $s_{0}=10^{4}$ (Petit, 1993; Williams and Wetherill, 1994). In other words, an asteroid of diameter $d$ can be destroyed by an impactor of diameter $d^{\prime} \geq 0.046 d$. The characteristic time $T_{c N}$ to destroy an asteroid of diameter $d \geq 1 \mathrm{~km}$ through a collision with a body of diameter $d^{\prime} \geq 100 \mathrm{~m}\left(s_{0}=10^{3}\right)$ equals 5 Gyr, and for $d=1 \mathrm{~km}$ and $d^{\prime}=46 \mathrm{~m}\left(s_{0}=10^{4}\right)$, it is $T_{c N} \approx 1 \mathrm{Gyr}$ (Ipatov, 1995b). These estimates are of the same order as those by Bottke and Greenberg (2001), who found that a 30-km asteroid is disrupted by some $\approx 2.5$ $\mathrm{km}$ projectile in 4.6 Gyr. Canavan (1993) obtained that $\alpha=3$ for NEOs with $d<40 \mathrm{~m}$ and $\alpha=2$ at $40 \mathrm{~m}$ $<d<2 \mathrm{~km}$. If we assume that values of $\alpha$ for the MAB are the same as those for NEOs, then at $s_{0}=10^{4}$ for $d=100 \mathrm{~m}\left(d^{\prime}=4.6 \mathrm{~m}\right), d=10 \mathrm{~m}\left(d^{\prime}=46 \mathrm{~cm}\right)$, and $d=1 \mathrm{~m}\left(d^{\prime}=4.6 \mathrm{~cm}\right)$, the values of $T_{c N}$ will be smaller by a factor of $40 / 4.64 \approx 8.6 ; 86$; and 860 , respectively, than those for $\alpha=2$. In this case, the collisional lifetime of a 1-m body is only about $1 \mathrm{Myr}$; this means that numerous meteoroids are debris of larger asteroid-like bodies. Some small cometary objects and NEOs can be also crashed during their travel via the MAB.

For the EKB (at $M_{E K B} \sim 0.1 m_{\oplus}$ ), the characteristic time $T_{c N}$ elapsed up to a collision of a body with diameter $d \geq D$ with some other body $d \geq D_{2}$ is greater by an order of magnitude than the values of $T_{c N}$ obtained for the MAB at the same values of $\alpha$ and $s_{0}=\left(D / D_{2}\right)^{3}$, but it is of the same order of magnitude for $D=1 \mathrm{~km}$ if we consider $10^{10} 1-\mathrm{km}$ and $10^{12} 100-\mathrm{m}$ TNOs. The energy of a collision is proportional to $v_{c}^{2}$, where $v_{c}$ is the relative velocity of a collison. For small bodies $v_{c}^{2} \propto\left(e^{2}+\sin ^{2} i\right) / a$, where $a, e, i$ are orbital elements. The value of $a$ is greater by a factor of 15 for the EKB than that for the MAB, a mean value of $\left(e^{2}+\sin ^{2} i\right)$ for the EKB is less by a factor of 1.4 than that for the MAB. So, for the EKB, $v_{c}^{2}$ is less by about a factor of $k \approx 20$, and for the same composition of two colliding bodies the ratio $s_{0}$ needed for destruction of a larger body will be smaller by a factor of $k$ than that for the MAB. Therefore, for the same composition of bodies, collisional lifetimes of TNOs are greater by about one or two orders of magnitude than those of asteroids. However, as it is more easy to destruct icy EKB bodies than rocky bodies in the $\mathrm{MAB}$, then the above ratio $s_{0}$ may be much larger for the $\mathrm{EKB}$, and the collisional lifetimes of small bodies in the EKB may be of the same order that those in the MAB. If some TNOs are porous, then it may be more difficult to destroy them than icy and rocky bodies. The total mass of SDOs moving in highly eccentric orbits between 40 and $200 \mathrm{AU}$ is considered to be of the same order or greater than $M_{E K B}$. The mean energy of a collision of a SDO with an EKBO is greater (probably, on average, by a factor of 4) than that for two colliding EKBOs of the same masses. Therefore, though SDOs spend a smaller part of their lifetimes at a distance $R<50 \mathrm{AU}$, the probability of destruction of an EKBO (with $30<a<50$ AU) by SDOs can be even more than that by EKBOs.

In contrast to the MAB, TNOs can get into the regions, from which they can migrate to orbits of planets, mainly due to gravitational interactions of with other TNOs, but not by collisions. The evolution of three 
gravitating bodies with masses equal to that of Pluto was investigated by Ipatov (1980) with the use of the spheres of action method (two two-bodies problems) and by Ipatov (1988) by numerical integration. The sphere of action (i.e., the Tisserand sphere) of radius $r_{s} \approx R\left(m / M_{\odot}\right)^{2 / 5}$ was used, where $R$ is a distance from the Sun, $M_{\odot}$ is the mass of the Sun, $m$ is the mass of an object. Basing on the results of numerical investigations of three and a hundred gravitating objects, Ipatov (1995a,b, 1998) made some appraisals of the evolution of a disk of TNOs. The used formulas for characteristic times elapsed up to close encounters are different from those for the Öpik's approach used by other scientists. The case of variable inclinations was also considered. We obtained that the characteristic time elapsed up to a close encounter (up to $r_{s}$ ) of two test EKBOs with characteristic masses of the observed EKBOs $\left(m \sim 5 \cdot 10^{-12} M_{\odot}\right)$ is equal to $7 \cdot 10^{10}$ yr. If there are about $7 \cdot 10^{4}$ EKBOs with $d \geq 100 \mathrm{~km}$, then during the last 4 Gyr a body with $a \approx 40$ AU takes part in about 3000 close (up to the Tisserand sphere) encounters with EKBOs with $d \geq 100 \mathrm{~km}$. The mean eccentricity of EKBOs could not reach its present value during the last 4 Gyr due to their mutual gravitational influence at the present mass of the EKB. Such growth could take place only for $M_{E K B}$ equal to several $m_{\oplus}$ (Ipatov, 1995a,b). The probability of that during the last 4 Gyr an EKBO with $d \geq 100 \mathrm{~km}$ collided with one of EKBOs of the same size equals to 0.005. If the number of EKBOs with $d \geq 10 \mathrm{~km}$ is greater than that with $d \geq 100 \mathrm{~km}$ by a factor of 100, then a probability of a collision during the last 4 Gyr of an EKBO with $d \geq 100 \mathrm{~km}$ with some EKBO with $d \geq 10 \mathrm{~km}$ equals to 0.15 . As the mean eccentricity of EKBOs is about 0.1, variations in $a$ for colliding bodies can exceed several AU. Probably, less than $1 \%$ of 100-km EKBOs had such large variations in $a$ due to collisions during the last 4 Gyr.

Our numerical estimates showed (Ipatov, 1995a) that the mean variation in $a$ at one close encounter is $\delta a \sim(1-3) \cdot 10^{-5} a$ for $m=5 \cdot 10^{-12} M_{\odot}$ and mean eccentricities and inclinations of the observed EKBOs. The variation in $a$ of an object located in the middle of the EKB probably is proportional to $\delta a \sqrt{N_{c}}$ (where $N_{c}$ is the number of close encounters of this object with large EKBOs) and usually does not exceed $0.1 \mathrm{AU}$ during the last 4 Gyr (all estimates are for the present mass of the EKB). For many objects at the inner part of the belt (at $a<39 \mathrm{AU}$ ), $a$ could vary more monotonously and could decrease by more than 1 AU during the last 4 Gyr. Such a decrease in $a$ may be a reason of that a region $(36 \leq a \leq 39$ AU) with small values of $e$ and $i$ is unpopulated, though, as it was shown by Duncan et al. (1995), it is dynamically stable under the gravitational influence of planets for the last 4 Gyr. At some very close encounters of EKBOs, variations in $a$ can be about several AU. Several percents of EKBOs could take part in such very close encounters during the last 4 Gyr (Ipatov, 1998). The number of such close encounters is larger by an order of magnitude than the corresponding number of collisions, and so the role of gravitational interactions in variations of orbital elements is larger than that of collisions. On average, during the last 4 Gyr a EKBO had several close (up to $r_{s}$ ) encounters with Pluto and changed its $a$ by $\sim 0.1 \mathrm{AU}$ at these encounters.

\section{Migration of Trans-Neptunian Objects under the Gravitational Influence of Planets}

Ipatov (1999), Ipatov and Henrard (2000) made computer runs of the evolution of orbits of some TNOs under the gravitational influence of planets. The symplectic RMVS integrator worked out by Levison and Duncan (1994) was used. The orbital evolution of one hundred test TNOs with various initial orbital elements was considered. Initial values of semimajor axes were varied from 35 to $50 \mathrm{AU}$. The considered time span usually equaled $20 \mathrm{Myr}$. In some runs it reached 100-150 Myr. In contrast to investigations by other authors, we were interested in the migration of TNOs not only to the orbits of Neptune or Jupiter, but further to the Earth's orbit. It was obtained in our runs that during evolution four former TNOs reached Jupiter's orbit and for two of them the perihelion distance decreased from 34 and 27.5 AU to 1.25 and 1.34 AU in 25 and $64 \mathrm{Myr}$, and these bodies were ejected into hyperbolic orbits in 30 and $70 \mathrm{Myr}$, respectively. As orbital elements were sampled with a time step equal to 20,000 yr, the actual minimal values of a perihelion distance can be smaller than those presented above.

Ipatov and Hahn (1999) investigated the evolution of orbits of Jupiter-crossing objects close to the orbit of the object $\mathrm{P} / 1996 \mathrm{R} 2$ and obtained $p_{J E} \approx 0.2$ and $\Delta t_{E} \approx 5000 \mathrm{yr}$, where $p_{J E}$ is the portion of Jupiter- 
crossing objects that reached the Earth's orbit during their lifetimes, $\Delta t_{E}$ is the mean time during which a Jupiter-crossing object crosses the Earth's orbit. At the initial step equaled to 30 days, the value of $p_{J E}$ was smaller by a factor of several for the use of the RMVS integrator than that for the integrator by Bulirsh and Stoer This difference was caused by the rare motion of bodies in the resonances, which decreased the perihelion distance, for the RMVS integrator. The use of the RMVS integrator could also cause an underestimate in the evaluation of probability for short-period comets to cross the Earth's orbit. Using this integrator, Levison and Duncan (1994) obtained about $0.5 \%$ of their total number for $\Delta t_{E}=10^{3}-10^{5} \mathrm{yr}$.

\section{The Number of Trans-Neptunian Objects Migrating to the Terrestrial Planets}

The number of TNOs migrating to the inner regions of the Solar System can be evaluated on the basis of simple formulas and the results of numerical integration. Let $N_{J}=P_{N} p_{J N} N$ be the number of former TNOs reaching Jupiter's orbit for the given time span $T$, where $N$ is the number of TNOs with $d \geq D$; $P_{N}$ is the fraction of TNOs leaving the belt and migrating to Neptune's orbit during $T$; and $p_{J N}$ is the fraction of Neptune-crossing objects which reach Jupiter's orbit for their lifetimes. Then the current number of Jupiter-crossers originated in the EKB equals $N_{J n}=N_{J} \Delta t_{J} / T$, where $\Delta t_{J}$ is the average time during which the object crosses Jupiter's orbit. According to Duncan et al. (1995), the fraction $P_{N}$ of TNOs that left the EKB during $T=4$ Gyr under the influence of the giant planets is $0.1-0.2$ and $p_{J N}=0.34$. As mutual gravitational influence of TNOs also takes place (Ipatov, 1998, 1999, 2000), we take this portion equal to 0.2 . Hence, taking $T=4 \mathrm{Gyr}, p_{J N}=0.34, \Delta t_{J}=0.2 \mathrm{Myr}$, and $N=10^{10}(d \geq 1 \mathrm{~km})$, we have $N_{J}=6.8 \cdot 10^{8}$ and $N_{J n}=3.4 \cdot 10^{4}$. Note that the number of Centaurs, coming from the EKB, turns out a bit less than $N_{C n}=P_{N} T_{C} N / T$, where $T_{C}$ is the mean Centaurs' lifetime. For $N=7 \cdot 10^{4}(d \geq 100 \mathrm{~km}), P_{N}=0.2$, $T=4 \mathrm{Gyr}$, and $T_{C}=10 \mathrm{Myr}$, this yields $N_{C n}=35$. The smaller $T_{C}$, the smaller $N_{C n}$. For Uranus-crossers $T_{C} \sim 10 \mathrm{Myr}$, although for the Chiron-type objects $T_{C} \sim 1 \mathrm{Myr}$.

We can now estimate migration of TNOs to the Earth. Let the number of bodies approaching the Earth's orbit during the considered time span $T$ be $N_{E}=N_{J} p_{J E}=P_{N} p_{J N} p_{J E} N$, and about $N_{J n} p_{J E} \Delta t_{E} / \Delta t_{J}$ former TNOs cross now both the orbits of Earth and Jupiter. Then the ratio of the number $N_{N E}$ of Earthcrossing objects (ECOs), which came from the EKB and are also Jupiter-crossers, to the total number $N_{E C O}$ of ECOs at the given time, is $P_{N E}=N_{N E} / N_{E C O}=P_{N} p_{J N} p_{J E} \Delta t_{E} N /\left(N_{E C O} T\right)$, where $N_{N E}=N_{E} \Delta t_{E} / T$. For $N=10^{10}(d \geq 1 \mathrm{~km}), N_{E C O}=750, T=4 \mathrm{Gyr}, p_{J E}=0.2$, and $\Delta t_{E} \approx 5000 \mathrm{yr}$, we have $P_{N E} \approx 0.2$, $N_{E} \approx 1.4 \cdot 10^{8}, N_{N E} \approx 170$, i.e. about $20 \%$ of 1 -km Earth-crossers can be former TNOs, which now cross both the orbits of Jupiter and Earth. For larger values of $\Delta t_{E}$, the values of $P_{N E}$ are greater. $N_{N E}$ is smaller by a factor of 2 for $N=5 \cdot 10^{9}$ (Jewitt and Fernandez, 2001) and it is much greater for $N=10^{11}$ (Jewitt, 1999) instead of $N=10^{10}$ as taken in the above estimate. Some TNOs with semimajor axes $a>50$ AU can also migrate to the orbits of Jupiter and Earth. So the number of former TNOs which now cross both the orbits of the Earth and Jupiter is greater than $N_{N E}$. Only a small part of Jupiter-crossing ECOs has been observed, because they use to move in distant orbits. Sometimes they move in 5:2, 7:3, 3:2 and other resonances with Jupiter (Ipatov and Hahn, 1999). As it is more easy to destruct icy bodies than stone or metal bodies (for example, on their way to the Earth, bodies can break up at close encounters with planets and at collisions with small bodies), the portion of TNOs among ECOs for bodies with $d \leq 100$ m (for example, for Tunguska-size bodies) may be greater than that for 1-km bodies, but small icy bodies disappear in the atmosphere and cannot reach the surface of the Earth.

If one denotes the fraction of Jupiter-crossers reaching Earth-crossing orbits with aphelion distance $Q \leq 4.5 \mathrm{AU}$ as $P_{Q}$, and their mean residence time in such orbits as $\Delta t_{E}^{*}$, then the number of such objects proves to be $N_{N E}^{*}=N_{E} P_{Q} \Delta t_{E}^{*} / T$. Valsecchi et al. (1995) obtained that the dynamical lifetime of comet $\mathrm{P} /$ Encke is about $0.35 \mathrm{Myr}$. The active lifetime of this comet is considered to be $\sim 10^{3}-10^{4} \mathrm{yr}$. Wetherill (1988) estimated that extinct comets could provide $40 \%$ of NEOs if one short-period comet evolved to an

Encke-type orbit every $5 \cdot 10^{4} \mathrm{yr}$. Using the spheres of action method, Ipatov (1995b) investigated the orbital evolution of initially Jupiter-crossing bodies under the gravitational influence of all planets and found that 
$P_{Q}$ is about 0.04 (in these runs, several bodies got $Q<1 \mathrm{AU}$ ). In the case of numerical integration, $P_{Q}$ is smaller. Harris and Bailey (1998) estimated $P_{Q}$ to be less than 0.0023. For about a hundred runs of evolution of Jupiter-crossing objects, Ipatov and Hahn (1999) didn't obtain Encke-type orbits. There is a possibility of decreasing $Q$ due to collisions with small bodies and destructions of comets during extremely close encounters with the terrestrial planets. Weissman (1994) considered that gas sublimation from the cometary nucleus can also decrease $Q$. Asher et al. (2001) showed that the rate at which objects may be decoupled from Jupiter and attain orbits like NEOs is increased by a factor of four or five $\left(P_{Q} \sim 0.011\right)$, producing $\sim 20 \%$ of the observed NEO population, if nongraviational forces are included in integrations as impulsive effects. For $N_{J n}=30000$ (i.e., $d \geq 1 \mathrm{~km}$ ), $\Delta t_{E}^{*}=0.35 \mathrm{Myr}$, and $\Delta t_{J}=0.2 \mathrm{Myr}$, we obtain $N_{E n}^{*} \approx 500$ for $P_{Q}=0.01$ and $N_{E n}^{*} \approx 100$ for $P_{Q}=0.002$. If a former Jupiter-crossing body moves in a typical Earth-crossing orbit, then $T_{E n}$ and so $N_{E n}$ can be even larger. Therefore the number of former TNOs which now move inside Jupiter's orbit may be of the same order (or even more) as the number of former TNOs now crossing both the orbits of Jupiter and Earth.

\section{Total Delivery of Celestial Bodies to the Earth}

Let us assess the potential collisions of celestial bodies with the terrestrial planets, taking the Earth as an example. The number of collisions of former TNOs, which now move in Jupiter- and Earth-crossing orbits, with the Earth during time $T$ equals to $N_{c o l}^{T N}=N_{E} \Delta t_{E} / T_{E}$, where $T_{E}$ is a characteristic time elapsed up to a collision of such ECO with the Earth. Note that the mean value of $T_{E}$ for the Jupiter-crossing ECOs is greater by a factor of several than that for other ECOs, so the portion of collisions of such objects is smaller than their portion among ECOs. Bottke et al. (1994) obtained a mean collisional lifetime $T_{E c}=134 \mathrm{Myr}$ for ECOs, and Ipatov (2000) using analytical formulas got $T_{E c}=100 \mathrm{Myr}$ for the observed 417 ECOs. Ipatov's estimate $T_{E c}=105 \mathrm{Myr}$ for 363 Apollo objects is in accord with $T_{E c}=120 \mathrm{Myr}$ calculated by Dvorak and Pilat-Lohinger (1999) on the basis of the numerical integration of evolution of 54 Apollo objects. At $e=0.7, a=3.06 \mathrm{AU}(P=5.35 \mathrm{yr})$, and $i$ varying from 0 to $30^{\circ}$, we have $T_{E}=400 \mathrm{Myr}$. Note that about half of observed Jupiter-family comets with $q<1$ AU have $P<5.35 \mathrm{yr}$ and for another half $P>5.35$ AU.

For $N=10^{10}(d \geq 1 \mathrm{~km}), T=4 \mathrm{Gyr}, p_{J E}=0.2, \Delta t_{E} \approx 5000 \mathrm{yr}$, and $T_{E}=400 \mathrm{Myr}$, we obtain $N_{c o l}^{T N} \approx 1750$, i.e., one collision per $2.3 \mathrm{Myr}$. For all ECOs with $d \geq 1 \mathrm{~km}$ at $N_{E C O}=750$ and $T_{E c}=100$ Myr, we have about 7.5 collisions per Myr. Therefore, the portion of former TNOs collided the Earth from Jupiter-crossing orbits among all 1-km bodies collided the Earth can be about several percents. The number of all 100-m ECOs is estimated to be about 70,000-160,000, so such objects collide the Earth on average ones in 600-1400 yr. For $d \geq 70 \mathrm{~m}$ the frequency of collisions is greater by a factor of 2 than that for $d \geq 100$ $\mathrm{m}$, and Tunguska-size objects collide the Earth once in several hundreds ( 500) years.

Following the generally accepted concept that about half of NEOs are ECOs, we shall assess the late delivery of NEOs and former TNOs to the Earth. We assume that the number of NEOs with radii between $r$ and $r+d r$ equals $n(r)=c d r / r^{3}$, where the dimension of $c$ is $r^{2}$. Integrating $n(r) \cdot(4 / 3) \pi \rho r^{3}=(4 / 3) c \pi \rho d r$ from 0 to $r_{\max }$, we obtain the total mass of ECOs $M_{e c o}=(4 \pi / 3) \rho c r_{\max }$, where $\rho$ is the mean density of NEOs and $r_{\max }$ is the radius of the largest NEO $(\sim 20 \mathrm{~km})$ or the largest ECO $(\sim 5 \mathrm{~km})$. Below we consider $r_{\max }=20 \mathrm{~km}$. Integrating $n(r)=c d r / r^{3}$ from some radius $r_{*}$ to $r_{\max }$, we obtain that the number $N_{*}$ of bodies with radius $r>r_{*}$ is $c k_{r} /\left(2 r_{*}^{2}\right)$, where $k_{r}=1-\left(r_{*} / r_{\max }\right)^{2}$. Obviously, for $r_{*} / r_{\max }=0.1$, $k_{r}=0.99 \approx 1$. For example, if $r_{*}=0.5 \mathrm{~km}$, the mass $m_{*}=(4 / 3) \pi \rho r_{*}^{3}=(\pi / 6) k_{\rho} 10^{15} \approx 5.2 \cdot 10^{14} k_{\rho} \mathrm{g}$, where $k_{\rho} \sim 2$ is the ratio of the density $\rho$ of a NEO to that of water. Then the total mass of bodies with $r>r_{*}$ turns out to be $M_{*}=(4 \pi / 3) \rho c\left(r_{\max }-r_{*}\right)$. On the other hand, if the mean mass of a body with $r>r_{*}$ equals $M_{*} / N_{*}$, it will exceed $m_{*}$ by the factor of $\left(2 r_{\max } / r_{*}\right) r_{\max } /\left(r_{\max }+r_{*}\right) \approx 2 r_{\max } / r_{*}$. For example, for $\sim 1500$ NEOs with $r>0.5 \mathrm{~km}$ it yields $M_{\text {eco }} \approx 750\left(2 r_{\max } / r_{*}\right) m_{*} \approx 6 \cdot 10^{4} m_{*} \approx \pi 10^{19} k_{\rho}$ g. If we suppose that $M_{\text {eco }}$ did not change significantly during $T=4 \mathrm{Gyr}$, we obtain the total mass of bodies that collided with the Earth during $T$ to be equal to $M_{E t}=\left(T / T_{E c}\right) M_{\text {eco }} \approx 40 M_{\text {eco }}$. Assuming further that about $k_{i}=20 \%$ of them were icy bodies, the total mass of water delivered to the Earth during the last 4 
Gyr turns out about $16 \pi 10^{19} \approx 5 \cdot 10^{20} \mathrm{~g}$, i.e., about $3.6 \cdot 10^{-4}$ of the mass of the Earth's hydrosphere.

\section{CONCLUSIONS}

Due to the gravitational influence of large trans-Neptunian objects and mutual collisions, some transNeptunian objects can get such orbits, from which they can be moved inside the Solar System under the gravitational influence of planets. The portion of former trans-Neptunian objects among Earth-crossing objects can be not small (up to $20 \%$ or even more).

\section{ACKNOWLEDGEMENTS}

This work was supported by the Belgian office for scientific, technical and cultural affairs (in 1999), the Russian Federal Program "Astronomy" (project 1.9.4.1), the Russian Foundation for Basic Research (project 01-02-17540), and INTAS (project 240 of INTAS Call 2000).

\section{REFERENCES}

Asher, D. J., M. E. Bailey, and D. I. Steel, The Role of Non-Gravitational Forces in Decoupling Orbits from Jupiter, in Collisional Processes in the Solar System, ASSL book, eds. M. Ya. Marov and H. Rickman, in press, 2001.

Bailey, M. E., and V. V. Emel'yanenko, Dynamical Evolution of Halley-Type Comets, Mon. Not. R. Astron. Soc. 278, 1087-1110, 1996.

Binzel, R. P., M. A Barucci, and M. Fulchignoni, The Origins of the Asteroids, Sci. Am., 265, 66-72, 1991. Britt, D. T., D. J. Tholen, J. F. Bell, and C. M. Pieters, Comparison of Asteroid and Meteorite Spectra Classification by Principal Component Analysis, Icarus, 99, 153-166, 1992.

Bottke, W. F., and R. Greenberg, The Impact Record on Asteroid Surfaces, Collisional Processes in the Solar System, ASSL book, eds. M. Ya. Marov and H. Rickman, in press, 2001.

Bottke, W. F., Jr., M. C. Nolan, R. Greenberg, and R. A. Kolvoord, Collisional Lifetimes and Impact Statistics of Near-Earth Asteroids, in Hazards Due to Comets and Asteroids, ed. T. Gehrels, pp. 337357, The University of Arizona Press, Tucson \& London, 1994.

Canavan, G. H., Value of Space Defenses, in Proc. Near-Earth Object Intercept Workshop, eds. G. H. Canavan, J. C. Solem, and J. D. G. Rather, pp. 261-274, Los Alamos, 1993.

Davis, D. R., and P. Farinella, Collisional Evolution of Kuiper Belt Objects, Icarus, 125, 50-60, 1997.

Dvorak, R., and E. Pilat-Lohinger, On the Dynamical Evolution of the Atens and the Apollos, Planet. Space Sci., 47, 665-677, 1999.

Duncan, M. J., and H. F. Levison, A Disk of Scattered Icy Objects and the Origin of Jupiter-Family Comets, Science, 276, 1670-1672, 1997.

Duncan, M. J., H. F. Levison, and S. M. Budd, The Dynamical Structure of the Kuiper Belt, Astron. J., 110, 3073-3081, 1995.

Eneev, T. M., On Possible Structure of Outer (Trans-Neptunian) Regions of the Solar System, Sov. Astron. Letters, 6, pp. 295-300 in Russian edition, 1980.

Farinella, P., R. Gonczi, Ch. Froeschlé, and C. Froeschlé, The Injection of Asteroid Fragments into Resonances, Icarus, 101, 174-187, 1993.

Fernández, J. A., On the Existence of a Comet Belt beyond Neptune, Mon. Not. R. Astron. Soc., 192, 481-491, 1980.

Gladman, B., and M. Duncan, On the Fates of Minor Bodies in the Outer Solar System, Astron. J., 100, 1680-1696, 1990.

Gladman, B., F. Migliorini, A. Morbidelli, V. Zappalà, P. Michel, et al., Dynamical Lifetimes of Objects Injected in Asteroid Belt Resonances, Science, 277, 197-201, 1997.

Harris, N. W., and M. E. Bailey, Dynamical Evolution of Cometary Asteroids, Mon. Not. R. Astron. Soc., 297, 1227-1236, 1998. 
Hartmann, W. K., Planetary Cratering 1. The Question of Multiple Impactor Populations: Lunar Evidence, Meteoritics, 30, 451-467, 1995.

Holman, M. J., and J. Wisdom, Dynamical Stability in the Outer Solar System and the Delivery of ShortPeriod Comets, Astron. J., 105, 1987-1999, 1993.

Hughes, D. W., and N. W. Harris, The Distribution of Asteroid Sizes and its Significance, Planet. Space Sci., 42, 291-295, 1994.

Ipatov, S. I., An Approximate Method for Analysis of the Mutual Gravitational Influence of Bodies in a Protoplanet Cloud. A Possible Evolution of Pluto's orbit, Preprint of Inst. of Applied Math., N 43, Moscow, in Russian, 33 p., 1980.

Ipatov, S. I., Accumulation and Migration of the Bodies from the Zones of Giant Planets, Earth, Moon, and Planets, 39, 101-128, 1987.

Ipatov, S. I., Computer Simulation of the Possible Evolution of the Orbits of Pluto and Bodies of the Trans-Neptunian Belt, Kinematics Phys. Celest. Bodies, 4, N 6, 76-82, 1988.

Ipatov S. I., Evolution of Initially Highly Eccentric Orbits of the Growing Nuclei of the Giant Planets, Sov. Astron. Letters, 17, 113-119, 1991.

Ipatov, S. I., Migration of Bodies in the Accretion of Planets, Solar System Research, 27, 65-79, 1993.

Ipatov, S. I., Gravitational Interaction of Objects Moving in Crossing Orbits, Solar System Research, 29, 9-20, 1995a.

Ipatov, S. I., Migration of Small Bodies to the Earth, Solar System Research, 29, 261-286, 1995b.

Ipatov, S. I., Migration of Kuiper-Belt Objects Inside the Solar System, in Planetary Systems — the Long View, Proc. 9th Rencontres de Blois (June 22-28, 1997), eds. L. M. Celnikier and Tran Thanh Van, pp. 157-160, Editions Frontières, Gif sur Yvette, 1998.

Ipatov, S. I., Migration of Trans-Neptunian Objects to the Earth, Celest. Mech. Dyn. Astron., 73, 107-116, 1999.

Ipatov, S. I., Migration of Celestial Bodies in the Solar System, Editorial URSS Publishing Company, Moscow, in Russian, 320 p., 2000.

Ipatov, S. I., and G. J. Hahn, Evolution of the Orbits of the P/1996 R2 and P/1996 N2 Objects, Solar System Research, 33, 487-500, 1999.

Ipatov, S. I., and J. Henrard, Evolution of Orbits at the 2:3 Resonance with Neptune, Solar System Research, 34, N 1, 61-74, 2000.

Jewitt, D., Kuiper Belt Objects, Annu. Rev. Earth. Planet. Sci., 27, 287-312, 1999.

Jewitt, D., J. Luu., and J. Chen, The Mauna Kea-Cerro-Tololo (MKCT) Kuiper Belt and Centaur Survey, Astron. J., 112, 1225-1238, 1996.

Jewitt, D., and Y. Fernandez, Physical Properties of Planet-Crossing Objects, in Colisional Processes in the Solar System, ASSL book, eds. M. Ya. Marov and H. Rickman, in press, 2001.

Kenyon, S. J., and J. X. Luu, Accretion in the Early Kuiper Belt. I. Coagulation and Velocity Evolution, Astron. J., 115, 2136-2160, 1998.

Kenyon, S. J., and J. X. Luu, Accretion in the Early Kuiper Belt. II. Fragmentation, Astron. J., 118, 1101-1119, 1999.

Levison, H. F., and M. J. Duncan, The Gravitational Sculpting of the Kuiper Belt, Astrophys. J., 406, L35-L38, 1993.

Levison, H. F., and M. J. Duncan, The Long-Term Dynamical Behavior of Short-Period Comets, Icarus, 108, 18-36, 1994.

Levison, H. F., and M. J. Duncan, From the Kuiper Belt to Jupiter-Family Comets: The Spatial Distribution of Ecliptic Comets, Icarus, 127, 13-23, 1997.

Levison, H. F., J. J. Lissauer, and M. J. Duncan, Modelling the Diversity of Outer Planetary Systems, Astron. J., 116, 1998-2014, 1998. 
Luu, J., B. G. Marsden, D. Jewitt, C. A. Trujillo, C. W. Hergenrother, J. Chen, and W. B. Offutt, A New Dynamical Class of Objects in the Outer Solar System, Nature, 387, 573-575, 1997.

Marsden, B. G., and D. I. Steel, Warning Times and Impact Probabilities for Long-Period Comets, in Hazards Due to Comets and Asteroids, ed. T. Gehrels, pp. 221-239, The University of Arizona Press, Tucson \& London, 1994.

Migliorini, F., P. Michel, A. Morbidelli, D. Nesvorny, V. Zappalá, Origin of Multikilometer Earth- and Mars-Crossing Asteroids: A Quantitative Simulation, Science, 281, 2022-2024, 1998.

Morbidelli, A., J. Chambers, J. I. Lunine, J. M. Petit, F. Robert, G. B. Valsecchi, and K. E. Cyr, Source Regions and Timescales for the Delivery of Water to the Earth, Meteoritics 8 Planetary Science, 35, 1309-1320, 2000.

Morbidelli, A., and D. Nesvorny, Numerous Weak Resonances Drive Asteroids Toward Terrestrial Planets Orbits, Icarus, 139, 295-308, 1999.

Nakamura, T., and H. Kurahashi, Collisional Probability of Periodic Comets with the Terrestrial Planets: an Invalid Case of Analytic Formulation, Astron. J., 115, 848-854, 1998.

Öpik, E. J., Small Bodies in the Solar System 1: Survival of Cometary Nuclei and the Asteroids, Adv. Astron. Astrophys., 2, 219-262, 1963.

Petit, J.-M., Modelling the Outcomes of High-Velocity Impacts Between Small Solar System Bodies, Celest. Mech. Dyn. Astron., 57, 1-28, 1993.

Rabinowitz, D., E. Bowell, E. Shoemaker, and K. Muinonen, The Population of Earth-Crossing Asteroids, in Hazards Due to Comets and Asteroids, ed. T. Gehrels, The University of Arizona Press, Tucson \& London, pp. 285-312, 1994.

Safronov, V. S., Evolution of the Protoplanetary Cloud and Formation of the Earth and Planets, Moscow: Nauka. Transl. Israel Program for Scientific Translation, NASA TTF-677, 1972.

Shoemaker, E. M., R. F. Wolfe, and C. S. Shoemaker, Asteroid and Comet Flux in the Neighborhood of Earth, in Global Catastrophes in Earth History, eds. V. L. Sharpton and P. D. Ward, Geological Soc. of America, Special Paper 247 (Boulder: Geological Soc. of America), pp. 155-170, 1990.

Shoemaker, E. M., P. R. Weissman, and C. S. Shoemaker, The Flux of Periodic Comets near Earth, in Hazards Due to Comets and Asteroids, ed. T. Gehrels, The University of Arizona Press, Tucson \& London, pp. 313-335, 1994.

Stern, S. A., Collisional Time Scales in the Kuiper Disk and Their Implications, Astron. J., 110, 856-868, 1995.

Stern, S. A., On the Collisional Environment, Accretion Time Scales, Architecture of the Massive, Primordial Kuiper Belt, Astron. J., 112, 1203-1211, 1996a.

Stern, S. A., Signatures of Collisions in the Kuiper Disk, Astron. Astrophys., 310, 999-1010, 1996b.

Stern, S. A., and J. E. Colwell, Accretion in the Edgeworth-Kuiper Belt: Forming 100-1000 km Radius Bodies at $30 \mathrm{AU}$ and Beyond, Astron. J., 114, 841-849, 1997.

Thommes, E. W., M. J. Duncan, and H. F. Levison, The Formation of Uranus and Neptune in the JupiterSaturn Region of the Solar System, Nature, 402, 635-638, 1999.

Torbett, M., and R. Smoluchowski, Chaotic Motion in a Primordial Comet Disk beyond Neptune and Comet Influx to the Solar System, Nature, 345, 49-51, 1990.

Trujillo, C. A., D. C. Jewitt, and J. X. Luu, Population of the Scattered Kuiper Belt, Astrophys. J., 529, pp. L103-L106, 2000.

Valsecchi, G. B., A. Morbidelli, R. Gonczi, P. Farinella, Ch. Froeschlé, Cl. Froeschlé, The Dynamics of Objects in Orbits Resembling that of P/Encke, Icarus, 118, 169-180, 1995.

Vilas, F., and M. E. Zolensky, Water, Water Everywhere: The Aqueous Alteration History Recorded in Meteorites, Meteorite!, 5, N 1, 8-10, 1999.

Weissman, P. R., The Comet and Asteroid Impact Hazard in Perspective, in Hazards Due to Comets and Asteroids, ed. T. Gehrels, pp. 1191-1212, The University of Arizona Press, Tucson \& London, 1994. 
Weissman, P. R., The Kuiper Belt, Annu. Rev. Astron. Astrophys., 33, 327-357, 1995.

Weissman, P. R., M. F. A'Hearn, L. A. McFadden, and H. Rickman, Evolution of Comets into Asteroids, in Asteroids II, eds. R. P. Binzel, T. Gehrels, and M. S. Matthews, pp. 880-919, Tucson: Univ. Arizona Press, 1989.

Wetherill, G. W., Where do the Apollo objects come from?, Icarus, 76, 1-18, 1988.

Wetherill, G. W., End Products of Cometary Evolution: Cometary Origin of Earth-Crossing Bodies of Asteroidal Appearance, In Comets in the Post-Halley Era, Proc. of the IAU Colloquium 121, eds. R. L. Newburn, J. Rahe and M. Neugebauer, pp. 537-556, Amsterdam: Kluwer Acad. Publ., 1991.

Williams, D. P., and G. W. Wetherill, Size Distribution of Collisionally Evolved Asteroidal Populations: Analytical Solution for Self-Similar Collision Cascades, Icarus, 107, 117-128, 1994.

Whipple, F. L., 1983 TB and the Geminid Meteors, IAU Circ., No. 3881, 1983.

Zharkov, V. N., and A. V. Kozenko, The Role of Jupiter in the Formation of the Giant Planets, Sov. Astron. Lett., 16, no 2, pp. 169-173 in Russian edition, 1990.

Zolensky, M. E., R. J. Bodnar, E. K. Gibson, Jr., L. E. Nyquist, Y. Reese, Chi-Yu Shih, H. Wiesmann, Asteroidal Water within Fluid Inclusion-Bearing Halite in an H5 Chondrite, Monahans (1998), Science, 285, 1377-1379, 1999. 